Original Paper http://ajol.info/index.php/ijbes http://indexmedicus.afro.who.int

\title{
Caractérisation de l'élevage porcin au Bénin
}

\author{
Abdou Wassiou TASSOU ${ }^{1 *}$, Sabbas ATTINDÉHOU ${ }^{1,2}$ et Sahidou SALIFOU ${ }^{2}$ \\ ${ }^{I}$ Unité de Recherche en Santé Animale et Biosécurité (URSAB), Laboratoire des Sciences Animales et \\ Halieutiques (LASAH), Ecole Doctorale des Sciences Agronomiques et de l'Eau (EDSAE), Université \\ Nationale d'Agriculture (UNA), Bénin. \\ ${ }^{2}$ Laboratoire Nationale de Parasitologie Vétérinaire (LNPV), Ecole Polytechnique d'Abomey-Calavi (EPAC), \\ Université d'Abomey-Calavi (UAC), Bénin. \\ ${ }^{*}$ Corresponding author, BP: 51 Parakou, Bénin; E-mail: tassabdou@yahoo.fr ; Tel.: +22966 547074
}

Received: $26-03-2021$ Accepted: $24-08-2021$

Published: $31-08-2021$

\section{RESUME}

L'agriculture béninoise subit depuis 2016 une mutation structurelle qui favorise la levée des contraintes notamment celles financières. Alors que le sous-secteur des productions végétales en tire déjà un grand profit affichant des performances record, l'élevage peine encore à faire sa révolution. La présente étude a pour objectif de faire la typologie de l'élevage porcin au Bénin et d'élaborer un plan de relance de la filière. Une revue de la littérature sur l'élevage porcin et une enquête typologique auprès de 308 éleveurs de porcs ont été réalisées d'Octobre 2019 à Février 2020. Les résultats ont montré que le porc, notamment celui de la race locale, est élevé dans toutes les contrées du Bénin, par tous les groupes socioculturels. L'activité parait bien secondaire pour la plupart (91\%) des éleveurs qui possèdent de petites unités de moins de vingt animaux dans $83 \%$ des cas. Le mode d'élevage en divagation (54,4\%) est le plus pratiqué et les risques sanitaires (peste et cysticercose) sont fréquemment évoqués pour expliquer la sous-production. Mais le défaut de technicité, de vocation et de financement semble être le véritable blocage. Une sensibilisation et/ou formation des éleveurs et des jeunes diplômés des écoles agricoles sur les nouvelles opportunités de développement de la filière devrait la dynamiser. (C) 2021 International Formulae Group. All rights reserved.

Mots clés : Porc, élevage, typologie, réforme, Bénin. 


\title{
Characterization of the pig farming in Benin
}

\begin{abstract}
Since 2016, Benin agriculture has undergone a structural change which favors the lifting of constraints, particularly financial ones. While the crop production sub-sector is already profiting greatly, showing record performance, livestock are still struggling to revolutionize. The objective of this study is to establish the typology of pig farming in Benin and to develop a revival plan for the sector. A review of the literature on pig breeding and a typological survey of 308 pig farmers were carried out from October 2019 to February 2020. The results showed that the pig, especially that of the local breed, is raised in all the regions of Benin, by all socio-cultural groups. The activity appears to be secondary for most (91\%) of breeders who own small units of less than twenty animals in $83 \%$ of cases. The method of rearing in straying $(54.4 \%)$ is the most practiced and health risks (plague and cysticercosis) are frequently mentioned to explain underproduction. But the lack of technicality, vocation and financing seems to be the real blockage. Awareness-raising and / or training of breeders and young graduates of agricultural schools on new development opportunities in the sector should boost it.
\end{abstract}

(C) 2021 International Formulae Group. All rights reserved.

Keywords: Pork, breeding, typology, reform, Benin.

\section{INTRODUCTION}

A la faveur d'importantes réformes structurelles et fonctionnelles, l'agriculture béninoise enregistre un embelli inédit marqué par une croissance du produit intérieur brut (PIB) de 5,5\% (Banque Mondiale, 2019 ; MEF, 2020). La production record de 678000 tonnes de coton en 2019 a hissé le pays au premier rang des producteurs de coton africain. Bien d'autres spéculations telles que l'ananas $\left(5^{\text {ème }}\right.$ producteur du continent) et l'anacarde font la fierté des acteurs. Il est donc évident que les productions végétales tiennent une part importante dans la croissance agricole du pays mais la contribution des productions animales ne suit vraisemblablement pas la même dynamique. Le marché de viande reste largement dominé par des importations massives de produits extérieurs (2 000 tonnes de poulets et 500 tonnes de porcs importés en 2018) avec une menace sans cesse croissante sur la sécurité et la sûreté alimentaire (Dognon et al., 2018). Certes, à la suite d'appuis subséquents les filières bovine, ovine, caprine et aviaire semblent décollées; mais la filière porcine reste irrésistiblement à la traine. La faible production porcine au niveau national, estimée à 504000 têtes de porcs en 2018 (FAOSTAT, 2020) contre les 2.500.000 têtes de bovins et 3.000 .000 de têtes de petits 
ruminants (DE, 2017), suscite des interrogations. La présente étude est initiée dans le but de mettre à jours les données caractéristiques du système de production ; de mettre en évidence les goulots d'étranglement et de proposer un plan technique de relance de la filière porcine.

\section{MATERIEL ET METHODES}

\section{Zone d'étude}

Les investigations conduites dans le cadre de ce travail ont été effectué dans toutes les zones agro écologiques (ZAE) du pays $\left(9^{\circ}\right.$ $\left.30^{\prime} \mathrm{N}, 2^{\circ} 15^{\prime} \mathrm{E}\right)$. L'enquête typologique a donc ciblé les acteurs de toutes ces zones avec une attention particulièrement orientée vers les communes de la partie méridionale et centrale réputées pour une production et/ou une consommation massive de porcs. La Figure 1 présente la zone d'étude constituée de trentetrois (33) communes.

\section{Recherche et analyse documentaires}

Dans le but de caractériser l'environnement institutionnel actuel de la gouvernance vétérinaire en général et de la gestion de l'élevage porcin en particulier, une enquête documentaire a été réalisée. Trois principales sources documentaires ont été exploitées. Il s'agit des archives du Ministère de l'Agriculture, de l'Elevage et des Pêches du Bénin (MAEP/Bénin), de celles de la Direction de l'Elevage du Bénin (DE/Bénin) et de la base de données FAOSTAT de l'Organisation des Nations Unies pour l'Alimentation et l'Agriculture (FAO). Secondairement, la production scientifique des universités béninoises et africaines ont été explorées pour élucider la typologie des activités de productions porcines. Ainsi, le Programme d'Actions du Gouvernement du Bénin, les directives, les rapports, les statistiques et les publications scientifiques portant sur l'élevage porcin au Bénin et en Afrique ont été consultés. Au total, huit (11) articles scientifiques, cinq (05) mémoires et thèses, sept (07) rapports techniques portant sur l'élevage porcin ont été consultés.

\section{Enquête typologique}

Une enquête transversale couplée avec des observations directes sur les élevages et les effectifs porcins a été réalisée. Un questionnaire structuré adressé aux éleveurs a permis de collecter les informations relatives à la taille et à la structure du troupeau, aux races élevées, au mode de conduite des animaux, au type d'habitat, au mode d'alimentation et au suivi sanitaire des animaux. La phase de collecte des données s'est déroulée dans les trente-trois communes cibles des douze (12) départements du pays. Au total, 308 élevages dont au moins 20 par département ont été identifiés au hasard pour la collecte des données. 


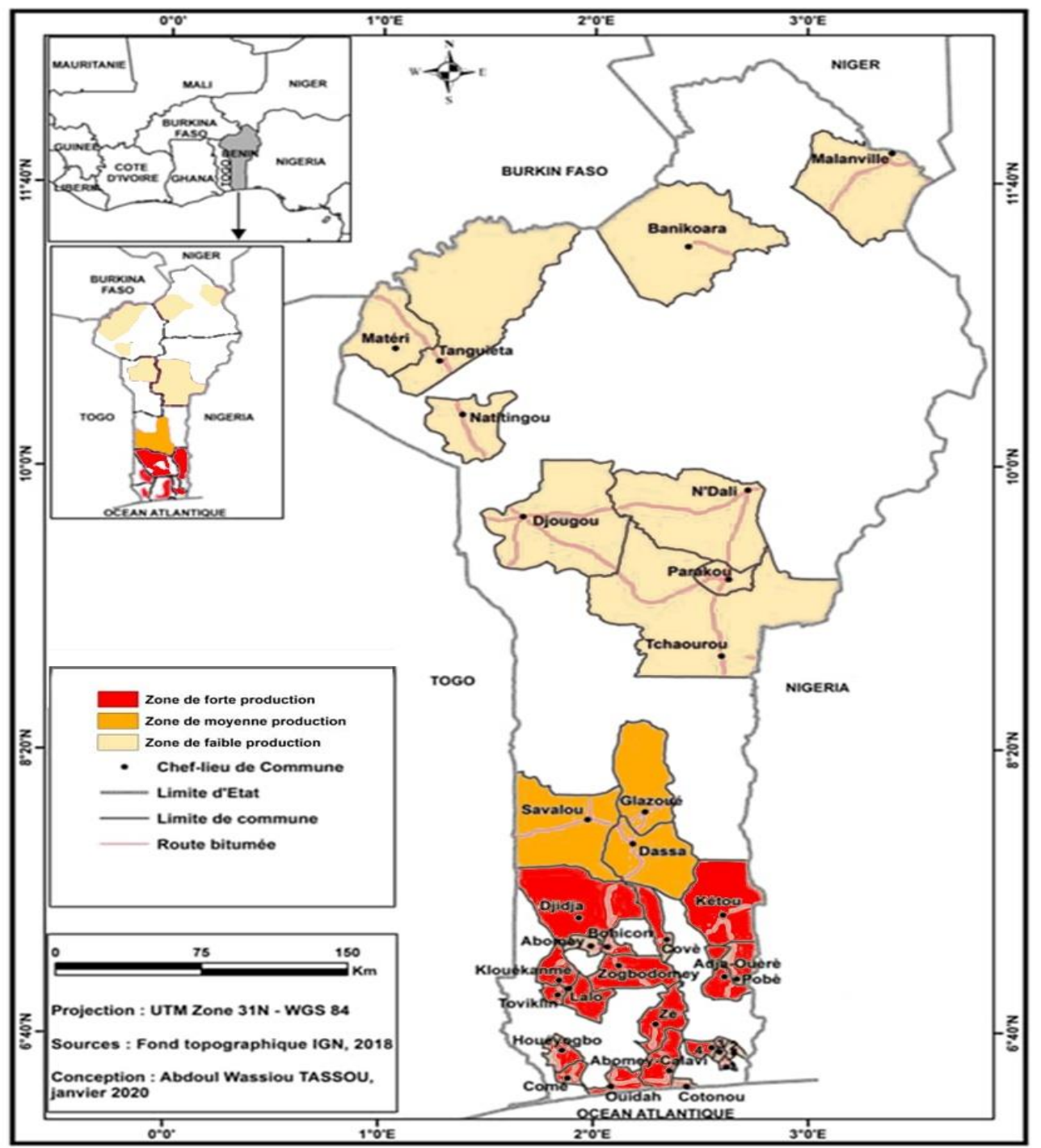

Figure 1: Zone d'étude pour la caractérisation de l'élevage porcin au Bénin.

\section{RESULTATS}

\section{Environnement institutionnel}

Au Bénin, la gouvernance vétérinaire est assurée par le ministère en charge de l'élevage. Elle implique plusieurs acteurs que sont l'Etat, les organisations professionnelles d'éleveurs et des structures privées. Le Ministère en charge de l'élevage est la principale structure chargée de l'administration et de la gestion de l'élevage au Bénin. Cette tâche est confiée à une direction technique, la Direction de l'Elevage (DE) qui travaille en collaboration avec les Agences Territoriales de Développement Agricole (ATDA) et les Directions Départementales de l'Agriculture, de l'Elevage et de la Pêche (DDAEP). La Direction de l'Elevage élabore et assure le suivi de l'exécution de la politique du gouvernement 
en matière d'élevage. Elle est chargée de définir la politique de l'Etat en matière de santé et de production animale, de santé publique vétérinaire et de veiller à son application. A cet effet, elle assure la protection sanitaire des animaux, diversifie et intensifie les productions animales et met en œuvre les conditions devant permettre un développement durable de l'élevage et son intégration à l'économie de marché. C'est aussi à la DE qu'incombe la sécurité alimentaire des populations rurales et urbaines par rapport aux denrées d'origine animale. Cette charge est assumée dans la pratique par quatre services techniques et un service administratif et financier. Sous la tutelle de la direction de l'élevage, plusieurs structures d'appui techniques et de nombreux projets de développement de l'Elevage œuvrent pour le bon accomplissement des missions. Quelques centres spécialisés constituent les bras techniques de l'administration publique. Il s'agit des fermes d'Etat (celle de l'Okpara créée en 1952 dans le département du Borgou ; celle de Kpinnou créée en 1958 dans le Mono et celles de Bétécoucou et de Samiondji créées respectivement en 1973 et 1975 dans le département des Collines); de l'abattoir de référence de Cotonou-Porto-Novo; des laboratoires vétérinaires de Bohicon et de Parakou; du Centre National des Produits Biologiques et Vétérinaires (CNPBV); etc.

Des organisations professionnelles (OP) agricoles en pleine émergence dans les divers sous-secteurs, des organisations non gouvernementales (ONG) ainsi que les collectivités locales s'affirment de plus en plus comme des acteurs incontournables dans la cogestion du sous-secteur élevage. Le secteur privé à travers des opérateurs individuels ou diverses associations professionnelles, jouent un rôle important dans le dispositif institutionnel de l'élevage, pour l'organisation des activités en amont et en aval de la production (approvisionnement en intrants, commercialisation, prestations de services, etc.). Dans la filière porcine, les éleveurs de porc sont organisés dans deux associations phares que sont l'Association Nationale des Eleveurs de Porcs (ANEP) et l'Association National des Traitants de porc (ANTP). Ces regroupements ont pour raison d'être la recherche d'appuis techniques ou financiers auprès des programmes de développement de l'élevage porcin ou des institutions de microfinance. Au Bénin, les organisations professionnelles (OP) sont fédérées en trois réseaux nationaux. On distingue la Fédération des Unions des Producteurs du Bénin (FUPRO) constituée de six Unions Régionales de Producteurs, le Groupement des Exploitants agricoles (GEA) et les Chambres d'Agriculture. Des partenaires techniques et financiers en l'occurrence la Banque Africaine de Développement (BAD) et le Fond Africain de Développement (FAD) participent à la vie du sous-secteur.

Depuis 2019, dans la dynamique des réformes institutionnelles diligentées par le gouvernement, un nouveau dispositif d'administration $\mathrm{du}$ domaine agricole et d'accompagnement des acteurs du monde rural est en vigueur. Ce dispositif marque une séparation des fonctions régaliennes de celles d'appui au développement des filières 
agricoles. Il répartit l'ensemble du territoire béninois en sept pôles de développement agricole (Tableau 1) en tenant compte des conditions agro-climatiques et des potentialités de chaque région. La coordination des pôles est assurée par le ministère de l'Agriculture, de l'Élevage et de la Pêche (MAEP), qui va créer des directions dans chaque département. À côté des sept pôles de développement, il est prévu sept agences territoriales de développement agricole et douze directions départementales de l'Agriculture, de l'Élevage et de la Pêche. L'Agence territoriale a pour mission de «mettre en œuvre la politique agricole et d'initier les actions propres à promouvoir les filières porteuses spécifiques au Pôle». La réussite de cette réforme du secteur passe par la cohérence et la rationalisation du nouveau cadre institutionnel du MAEP. A défaut de la promotion et/ ou de la création de banques purement agricoles tant souhaitées par les exploitants agricoles, l'État a institué un Fonds National de Développement Agricole crédité de cent milliards de francs CFA (soit 180.664.421 USD) servant de garantis auprès des institutions financières pour des prêts bancaires (Programme d'Action du Gouvernement, 2016).

\section{Répartition géographique des exploitations, effectifs et races de porcs au Bénin}

L'élevage porcin est assez développé et répandu au Bénin. Des exploitations porcines ont été enregistrées dans tous les départements du pays sans exception (Tableau 2). La FAO a estimé le cheptel national à 504000 têtes de porcs en 2018 (FAOSTAT, 2020). L'effectif moyen dans les élevages a été de 13 têtes avec un minima de 01 tête et un maximum de 53 têtes. Il convient de relever que le cheptel national porcin a connu un développement rapide vers les années 1987 pour atteindre 436 860 têtes. Il est ensuite passé à 581600 têtes en 1996 avant d'être victime pour la première fois en 1997 d'une épizootie de peste porcine africaine (PPA) qui a ravagé plus de $70 \%$ du cheptel (DE Bénin, 2017). Depuis 2000, on observe une augmentation progressive de l'effectif du cheptel (Figure 2) qui est passé de 398000 têtes en 2012 à plus de 448360 en 2016 (FAOSTAT, 2017).

En termes de races élevées, le Tableau 2 présente les proportions relatives dans les douze départements. On a noté une propension à l'élevage des races améliorées dans les départements du sud Bénin comparativement aux races de porcs élevées au nord. Il a également été constaté que certains éleveurs gardaient au sein d'un même élevage plusieurs races de porcs différents pour l'amélioration de leurs cheptels. Il ressort de l'analyse de ce Tableau qu'au Bénin, l'élevage porcin est encore essentiellement basé sur les ressources génétiques endogènes (races locales). Le porc de race locale (Figure 3) se caractérise par un groin allongé avec un chanfrein rectiligne, de petites oreilles à port dressé ou port horizontal. Le corps est étroit et recouvert de soies longues et grossières. La peau est souvent noire, parfois pie mais rarement blanche. La robe noire est très caractéristique du porc local d'Afrique et peut constituer le premier critère phénotypique de tri de porc susceptible d'être de race locale pure parmi des animaux marqués par des 
croisements observés un peu partout en Afrique (Agbokounou et al., 2016). Au Bénin, le porc local a une portée moyenne de 6,86 et le poids moyen d'un porcelet est de $535 \mathrm{~g}$ à la naissance et $4550 \mathrm{~g}$ au sevrage, son gain moyen quotidien à l'engraissement varie de $74 \mathrm{~g} / \mathrm{j}$ à 126,4 g/j (Youssao et al., 2009a) en élevage amélioré et $60 \mathrm{~g} / \mathrm{j}$ en élevage traditionnel (Atodjinou et Dotcho, 2006). Le rendement de la carcasse est de $61,81 \%$ pour les femelles, $60,56 \%$ pour les mâles castrés et $57,7 \%$ pour les mâles entiers (Youssao et al., 2008). Le porc local a une bonne résistance à la chaleur et à l'insolation. Il a également une grande tolérance aux irrégularités alimentaires, au manque de soins de santé et il manifeste parfois une bonne fécondité (entre 10 à 12 porcelets par portée). Quelques races exotiques originaires d'Europe et de Chine complètent la population génétique porcine. $\mathrm{Ce}$ sont notamment le Large White, le Landrace et le MeiShan qui alimente un métissage très actif au sein de la population porcine.

\section{Typologie de l'élevage porcin au Bénin}

Classiquement, l'élevage du porc est pratiqué suivant trois modalités que sont, le mode extensif dit traditionnel, le mode semiintensif et le mode intensif. Au Bénin il convient de classifier cette production suivant deux modes tenant compte du système d'habitat et ou de contrôle du mouvement des animaux. Ainsi, on distingue l'élevage en claustration dans lequel les animaux sont gardés dans des habitats clos et où des aliments leurs sont apportés et l'élevage en divagation caractérisé par l'inexistence d'habitat proprement dit et dans lequel les animaux sont en liberté, recherchant par eux-mêmes des aliments (Figure 3a). Cette dernière modalité est significativement dominante car pratiquée par 54,4\% des éleveurs. Elle concerne surtout la production de porcs locaux (61\%) pour laquelle l'alimentation est assurée à $82,16 \%$ au moyen des restes de cuisine (Tableau 3). L'élevage porcin est majoritairement pratiqué par les hommes $(74,4 \%)$ pour qui, il représente une activité secondaire. Il en est ainsi pour $91 \%$ des éleveurs dans leur ensemble. Au plan sanitaire, le recours aux soins vétérinaires est assez rare. Seulement 39,6\% des éleveurs ont assuré y avoir recours. Selon les travaux de Houndonougbo et al. (2012) dans le Sud Est du pays, 46,7\% des éleveurs sont des jeunes ayant une tranche d'âge comprise entre 25 et 40 ans. Selon les mêmes auteurs, la main d'œuvre utilisée est essentiellement familiale et la majeure partie du financement des activités de production est sur fonds propres du producteur. La non professionnalisation de l'activité et la limitation à l'autofinancement expliqueraient en partie la sous-optimisation de la production. Presque $83 \%$ des élevages sont de petite taille (moins de 20 porcs).

\section{Performances zootechniques des porcs élevés au Bénin}

En termes de production, le porc local béninois présente des performances de croissance modestes et une faible productivité numérique, mais il est caractérisé par une grande rusticité. L'âge à la première saillie est de 6 mois avec une portée moyenne de 6,86 porcelets. Le poids à la naissance d'un porcelet 
local au Bénin varierait de 535,01 g à $810 \mathrm{~g}$ (Youssao et al., 2009a ; Youssao et al., 2009b). Quant aux races exotiques telles que les Landrace et Large White, elles ont un gain quotidien moyen qui varie entre 600 et $900 \mathrm{~g} / \mathrm{j}$ et une taille moyenne de portée qui varie entre 10 et 12 porcelets (Youssao, 2007). Le poids à la naissance rapporté pour le Large White a été de 886,4 g au Bénin (Youssao et al., 2009a). Les porcs améliorés en croissance expriment généralement un gain de poids allant de 750 à 950 g par jour.

\section{Situation sanitaire des élevages porcins au Bénin}

En élevage porcin au Bénin, les pathologies dominantes et fréquemment signalées par les éleveurs sont la gale, la cysticercose, les helminthiases, la toux, l'amaigrissement des porcelets, la diarrhée, les arthrites et la peste porcine africaine (Soglohoun, 2017). La présente étude a permis de relever que $60,4 \%$ des éleveurs n'appliquent pas de suivi sanitaire contre $39,6 \%$ qui font un suivi sanitaire rigoureux de leurs élevages porcins. Le mode de traitement dépend généralement du type d'élevage. Dans les élevages de type intensif et semi intensif les éleveurs font recours aux services vétérinaires. Les traitements appliqués sont le déparasitage, l'antibiothérapie et les supplémentations vitaminées. Dans les élevages traditionnels, la majorité des éleveurs n'accorde aucun soin à leurs animaux. L'utilisation de produits vétérinaires ou l'appel à un vétérinaire est la pratique des zones urbaines. Des savoirs endogènes basés sur les connaissances floristiques sont couramment évoqués comme moyens de lutte contre les pathologies.

Au nombre des pathologies porcines, la Peste Porcine Africaine (PPA) et la cysticercose ont une importance médicale et économique particulière. Apparue pour la première fois au Bénin en août 1997 sur la rive du lac Nokoué à Cotonou, la PPA s'était répandue de septembre à novembre 1997 dans plusieurs localités des départements de l'Atlantique, de l'Ouémé, du Mono et du Zou faisant perdre au pays plus de la moitié de son cheptel porcin (450 000 porcs sur un total de 600000 têtes) (Ayssiwede, 2004). Les différentes mesures de lutte ont permis d'aboutir à une accalmie en novembre 1998. Des foyers de résurgence ont vu le jour respectivement en mars et en septembre 2000 dans l'Atlantique et l'Ouémé, dans le Mono et le Zou. En 2001, la région septentrionale a connu sa première épizootie. La PPA reste à ce jour une menace. Quant à la cysticercose porcine, assez bien connue des éleveurs, elle est une véritable enzootie. Elle fait partie des principaux motifs de saisie de viande post inspection.

\section{Mode de commercialisation}

La filière porcine s'organise autour de divers produits, du porc sur pieds jusqu'à sa transformation en viande. La viande de porc est fortement demandée. Le mode et les techniques d'élevage du porc n'influencent pas sa commercialisation. Aussi bien les porcs issus des élevages traditionnels que des élevages semi-intensifs et intensifs sont écoulés sur le marché aussitôt après production. La 
commercialisation du porc se fait soit sur pied ou soit préparé (viande). La vente se fait suivant diverses modalités. Il y a la vente à domicile, la vente publique, la vente contractuelle et la vente de porcs transformés. La vente à domicile est le mode le plus répandu. Il fait l'objet de marchandage par des commerçants de porcs, des courtiers, des charcutiers, des consommateurs particuliers qui s'adressent directement à l'éleveur. Dans le système de ventes publiques, les porcs sont conduits aux marchés, où ils sont vendus sur pieds, à la criée, au plus offrant. Le marché de porcs sur pied est animé par deux principales catégories de commerçants que sont les vendeurs (producteurs/éleveurs et marchands de porcs sur pied) et les acheteurs (producteurs, marchands de porcs sur pied, bouchers, rôtisseurs, etc.) Le paiement des porcs se fait généralement au comptant au moment de l'enlèvement des porcs. Pour les marchands de bétails ou les charcutiers qui ont des liens anciens avec l'éleveur, le règlement des sommes dues n'intervient qu'une fois les porcs vendus par le charcutier, généralement au bout d'une à deux semaines. Il est surtout pratiqué par les marchands de porcs et les courtiers (Ayssiwede, 2004). Le système de ventes contractuelles est un système dans lequel les éleveurs ont la possibilité de passer un contrat avec un abattoir ou un centre moderne de transformation de porcs pour la livraison d'un certain nombre d'animaux à un prix donné sur une période déterminée. Ce système est destiné surtout aux élevages de grande taille et permet aux producteurs de planifier leur production à plus long terme. Il est très rarement pratiqué par les éleveurs, faute de centre moderne de transformation de porcs au Bénin. Enfin, la vente de porcs transformés correspond à un système dans lequel les animaux sont abattus et commercialisés sous forme de viande fraîche ou cuite (frite, rôtie ou braisée).

\section{Contraintes technico-économiques}

L'élevage porcin est soumis à de nombreuses contraintes techniques. Ainsi, au niveau national il n'existe pas de réseaux de diffusion des géniteurs performants pouvant aider les petits éleveurs à améliorer les races élevées. Dans un système d'élevage le plus souvent traditionnel, très peu d'intrants de bonne qualité sont utilisés dans l'alimentation des porcs. Ces derniers, déjà insuffisamment nourris et exposés aux intempéries diverses sont au mieux sous productifs. L'utilisation exclusive des sous-produits agricoles et agroindustriels observée dans la plupart de ces élevages, les coûts élevés, la pénurie de certaines matières premières et la concurrence avec l'homme particulièrement pour les céréales et les tubercules accentuent le problème de déséquilibre alimentaire tant quantitatif que qualitatif et constituent un handicap à la production. Le manque de suivi, d'encadrement et de formation des producteurs de porcs, de distribution des intrants et de commercialisation sont autant de freins à l'augmentation de la production et dénote de l'absence d'une politique de développement pour cette spéculation (Ayssiwede, 2004). L'insuffisance des ressources financières constitue elle aussi, un handicap au développement de l'élevage porcin au Bénin. 
Tableau 1: Descriptif des sept pôles de développement agricole au Bénin.

\begin{tabular}{cll}
\hline Pôles $\left(\mathbf{N}^{\circ}\right)$ & Aire géographique & Spécialisation culturale \\
\hline 1 & Vallée du Niger & Riziculture \\
& Sud Alibori / Nord Borgou / & Culture du coton \\
& Kandi - Kérou - Péhunco & Culture du coton \\
& Sud Borgou / Ouest Atacora / Donga & Cultures vivrières \\
3 & & Culture du coton \\
& Collines & Cultures vivrières \\
4 & & Culture d'anacarde \\
& & Diversifiée \\
5 & Zou et Couffo & Arboriculture \\
7 & Plateau & Culture du palmier à huile \\
& Ouémé / Atlantique / Mono & Pêcherie et maraichage \\
\hline
\end{tabular}

Source : PSDSA, 2025 ; PNIASAN 2017-2021

Tableau 2: Fréquence relative des différentes races de porcs dans les élevages au Bénin.

\begin{tabular}{lcc}
\hline \multirow{2}{*}{ Départements } & \multicolumn{2}{c}{ \% Races exploitées } \\
\cline { 2 - 3 } & Locale & Améliorée \\
\hline Alibori & 80 & 20 \\
Atacora & 57,4 & 42,6 \\
Atlantique & 68,6 & 31,4 \\
Borgou & 54,5 & 45,5 \\
Collines & 28,2 & 71,8 \\
Couffo & 94,5 & 5,5 \\
Donga & 93 & 7 \\
Littoral & 14 & 86 \\
Mono & 85,9 & 14,1 \\
Ouémé & 41,2 & 58,8 \\
Plateau & 69,8 & 30 \\
Zou & 43,3 & 56,7 \\
\hline
\end{tabular}


A. W. TASSOU et al. / Int. J. Biol. Chem. Sci. 15(4): 1338-1354, 2021

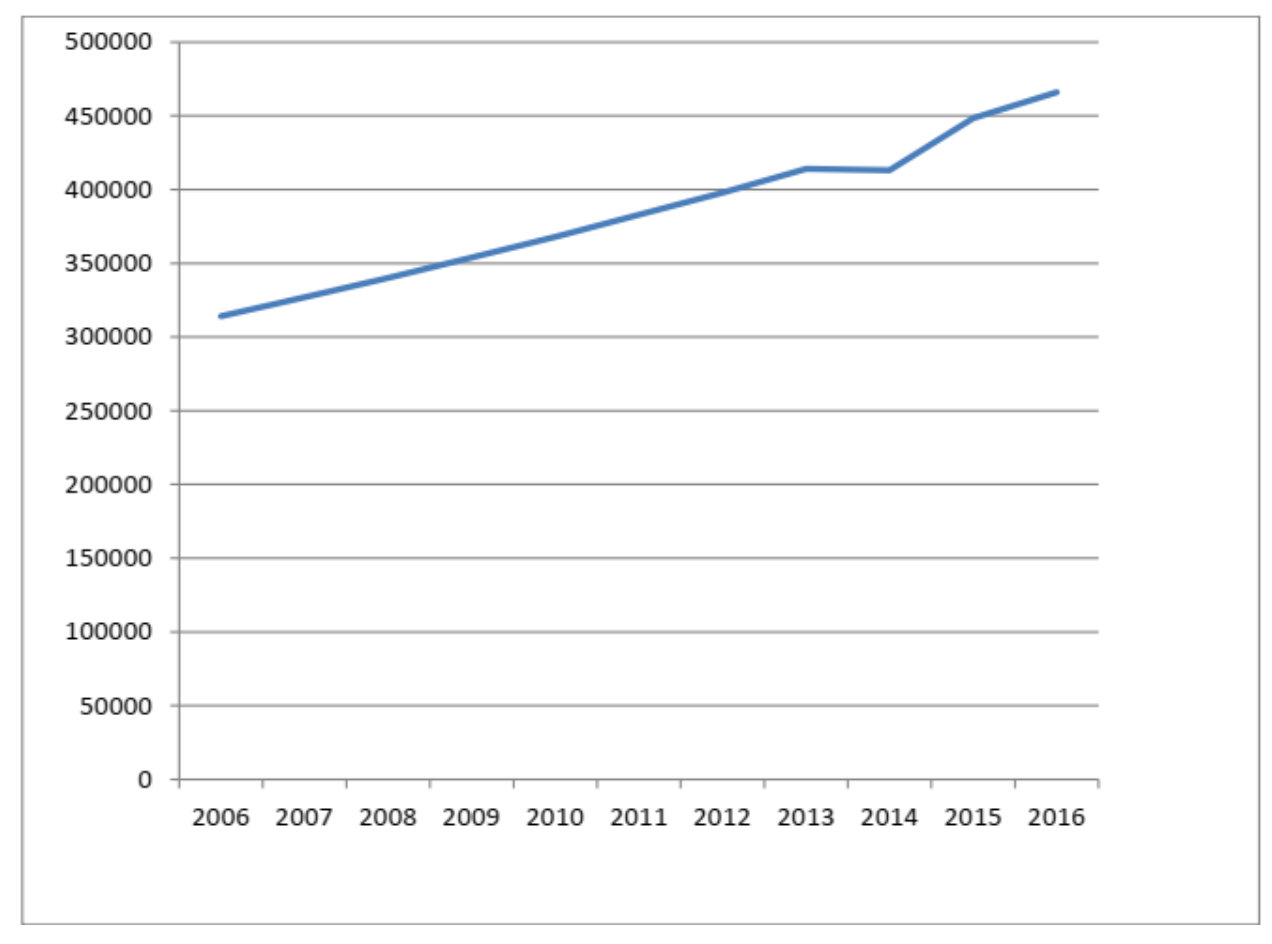

Figure 2: Evolution du cheptel porcin béninois entre 2000 et 2016.
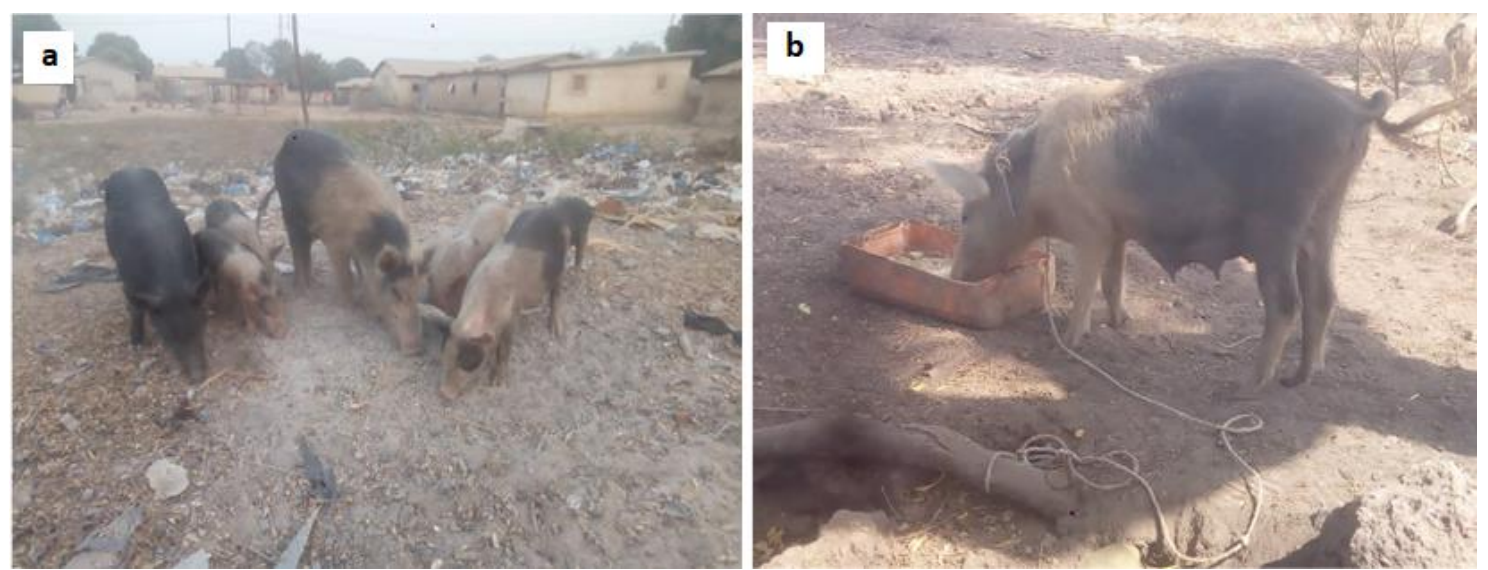

Figure 3: Photographie de porcs de race locale en divagation (a) et au piquet (b). 
Tableau 3: Eléments de typologie de la production porcine au Bénin.

\begin{tabular}{|c|c|c|}
\hline Variables & Catégories & $\begin{array}{c}\text { Pourcentage } \\
\text { (avec Chi } 2=\mathbf{2 , 2 8} \\
\text { et } \mathrm{P}<\mathbf{0 , 0 5} \text { ) }\end{array}$ \\
\hline \multirow{2}{*}{ Mode d'élevage } & Divagation & 54,4 \\
\hline & Claustration & 45,6 \\
\hline \multirow{2}{*}{$\begin{array}{l}\text { Taille des exploitations } \\
\text { (nombre } \mathrm{N} \text { de porcs dans }\end{array}$} & $\mathrm{N} \leq 20$ & 82,8 \\
\hline & $20<\mathrm{N} \leq 50$ & 15,9 \\
\hline l'élevage) & $\mathrm{N}>50$ & 1,30 \\
\hline \multirow{2}{*}{ Races porcines élevées } & Locale & 61 \\
\hline & Améliorée & 39 \\
\hline \multirow{2}{*}{ Genre des éleveurs (sexe) } & Homme & 74,4 \\
\hline & Femme & 26,6 \\
\hline \multirow{3}{*}{$\begin{array}{l}\text { Importance de l'élevage } \\
\text { porcin }\end{array}$} & Activité principale & 9 \\
\hline & Activité secondaire & 91 \\
\hline & Provende & 13,66 \\
\hline \multirow{2}{*}{ Mode d'alimentation } & Restes de cuisine & 82,16 \\
\hline & $\begin{array}{l}\text { Provende }+ \text { restes de } \\
\text { cuisine }\end{array}$ & 4,2 \\
\hline \multirow[t]{2}{*}{ Suivi sanitaire } & Oui & 39,6 \\
\hline & Non & 60,4 \\
\hline
\end{tabular}

\section{DISCUSSION}

\section{Environnement institutionnel}

Depuis 2016, avec la mise en œuvre de nouvelles réformes institutionnelles dans le secteur de l'élevage, une dynamique s'est instaurée. Ainsi, ce secteur s'est positionné comme l'un des leviers de développement économique du pays, de création de richesses et d'emplois. Avec les ATDA qui pilotent les filières les plus porteuses de leur pôle, l'amélioration des prestations au niveau des structures d'appui est devenue une réalité. Le contrôle est devenu plus systématique. Les normes techniques sont élaborées par les DDAEP et les suivies sont beaucoup plus rapprochées. Ces réformes ont permis de mieux adapter le mode d'élevage des animaux à cycle court (volailles, lapins, porcins, petits), des bovins, et le mini élevage non-conventionnel au contexte socioéconomique pour accroître les niveaux de production de viande tout en réduisant les coûts de production et d'assurer l'émergence des filières viandes porteuses afin d'accroître l'accès du pays aux devises. 
Répartition géographique des exploitations, effectifs et races de porcs au Bénin

Les données collectées à l'issue de l'enquête montrent que l'élevage des porcs est une activité assez développée et menée dans tous les départements du Bénin. Ceci a déjà été rapporté par Youssao (2015) qui a précisé la forte concentration des exploitations dans le Sud et le Centre du pays (Atlantique, Mono, Ouémé, Zou). Les exploitations de porcs rencontrées au cours des enquêtes étaient en majorité de petites tailles constituées en moyen d'un effectif de 13 têtes. Cet effectif moyen est inférieur à celui obtenu par Djimenou et al. (2017) au sud du Bénin (23 têtes), par Youssao et al. (2008) en zone périurbaine de Cotonou et d'Abomey-Calavi (19 têtes) et supérieur à celui obtenu par Houndonougbo et al. (2012) dans la commune d'Adjarra au Sud-Est du Bénin (11,4 têtes). Cette différence observée pourrait s'expliquer par la taille de l'échantillon. En effet, la présente étude a été conduite dans tous les départements du pays comparativement aux autres auteurs dont les enquêtes se sont déroulées dans une région donnée.

La présente étude a révélé que $61 \%$ des porcs élevés au Bénin sont de races locales. Ceci a été rapporté par Djimenou et al. (2017) et Dahouda et al. (2019) qui ont montré que l'élevage de porcs locaux représente en moyenne $58 \%$ et $56,5 \%$ du cheptel porcin au Sud du Bénin. Cependant, on observe une propension de l'élevage des races améliorées dans la partie méridionale et centrale du pays à différence de la partie septentrionale ou la race locale prédomine. Ce même constat a été fait par Djimenou et al. (2017) qui ont montré que l'élevage de porc local a connu une régression de 35,5\% de 1994 à 2015 au profit de l'élevage des croisés et des races exotiques dans le sud du Bénin. Dans le septentrion à majorité musulman, le tabou alimentaire qui frappe le porc a sans doute un effet régressif global sur le développement de l'élevage porcin.

\section{Typologie de l'élevage porcin au Bénin}

Le mode d'élevage extensif ou traditionnel est le plus répandu au Bénin avec pour principale caractéristique la divagation des porcs. Dans la présente étude, la divagation est la plus pratiquée $(54,4 \%)$. Ce résultat est conforme à ceux obtenus par Kiki et al. (2018) et Dahouda et al. (2019) qui ont observé de fortes proportions des élevages porcins en divagation dans le sud du Bénin.

L'étude a révélé que l'alimentation est assurée à 82,16\% au moyen des restes de cuisine. Ce résultat est conforme à celui obtenu par Soglohoun (2017) et Kiki et al. (2018) qui ont rapporté que les aliments les plus servis aux porcs sont des sous-produits agricoles ou agroalimentaires comme les déchets de cuisine, les épluchures de manioc et de légumes, des eaux basses de récupération d'huile de palme et d'autres aliments retrouvés par les animaux eux-mêmes en divagation.

Au plan sanitaire, le recours aux soins vétérinaires sont assez rares. Les enquêtes ont relevé que $60,4 \%$ des éleveurs n'appliquent pas de suivi sanitaire dans leurs élevages. La pratique du suivi sanitaire dépend généralement du type d'élevage. Dans les élevages de type intensif et semi intensif les éleveurs font recours aux services vétérinaires. 
Cela renforce le constat de Ayssiwede (2004) d'après qui l'élevage porcin ne bénéficie que d'une faible prise en charge sanitaire. Ceci pourrait s'expliquer par la taille réduite des exploitations et sans doute parce que l'activité passe au second plan pour la plupart des pratiquants. En effet, elle est l'apanage d'hommes pour qui elle représente une activité secondaire. Une proportion voisine de $95 \%$ avait été estimée par Djimenou et al. (2017) dans les départements du sud du Bénin. Du fait de la prédominance du système de production traditionnel avec des animaux divagants associé au manque de suivi et d'encadrement des producteurs en matière de santé animale, on assiste à la prolifération de plusieurs maladies dans les élevages porcins dont les plus courant sont les maladies parasitaires (gale, la cysticercose), la toux, l'amaigrissement des porcelets, la diarrhée et la PPA dont persiste encore quelques foyers. Ces observations sont conformes à celles rapportées par Soglohoun (2017) dans son étude sur les inventaires des pathologies porcines dans les départements de l'Ouémé et du Plateau au Bénin. Aussi, certaines pratiques favorisent la propagation des maladies. C'est le cas par exemple dans la partie septentrionale du Bénin à majorité musulmane, où, du fait des considérations religieuses la présence des porcs dans les abattoirs n'est pas tolérée et rien n'est vraiment mis en œuvre pour l'amélioration des conditions d'abattage et de préparation des carcasses de porc. Les animaux sont alors abattus et préparés aux domiciles ou dans les points de vente. Les carcasses sont ensuite présentées aux services d'inspection sur place ou dans des lieux indiqués. De telles pratiques laissent la porte ouverte à toute sorte de dissimulation de carcasses suspectes et même à d'éventuelle dissémination des agents pathogènes.

\section{Perspectives de développement de l'élevage porcin au Bénin}

L'élevage porcin a un fort potentiel de croissance et est appelé à jouer un rôle important dans l'accroissement rapide de la production de protéines animales (Ndébi et al., 2009). Au Bénin, il constitue une activité secondaire procurant de revenus supplémentaires à plusieurs couches socioprofessionnelles (Agbokounou et al., 2016). Plusieurs projets programmes ont été mis en œuvre par le gouvernement notamment le Programme National de Relance de l'Élevage du Porc (PNREP), et le Projet d'appui aux filières lait et viande (PAFILAV), le Projet de Développement des Filières Lait et Viande et de Promotion d'Entreprises d'Elevage (PRODEFILAV-PEL) pour l'amélioration de la production porcine par métissage. Également des programmes universitaires sont mis en œuvre à travers le projet de Professionnalisation et Renforcement de la Compétitivité de la Filière Porc par la Recherche Action en Partenariat dans les départements de l'Ouémé et du Plateau au SudEst du Bénin (ProficPorc) pour l'amélioration des capacités techniques des acteurs de la filière porc et la biosécurité des élevages. Tous ces projets ont eu pour objectifs respectifs de relancer l'élevage porcin après la Peste Porcine Africaine (PPA) et d'accroître la production et 
l'efficacité des secteurs bovin, ovin, porcin et laitier en fournissant des produits de qualité et en augmentant les revenus des parties prenantes. Ils s'articulent autour de la lutte contre la PPA, la gale, la cysticercose et l'amélioration génétique.

Quant aux contraintes économiques et financières, la garantie bancaire offerte par le Fonds National de Développement Agricole devra suffire à booster la production par l'accès plus facile au crédit puis à une amélioration technique de la production. Il reste que les éleveurs soient bien informés sur le mécanisme de développement entrepreneurial et sur la bonne gestion des crédits. Il faudra par ailleurs l'expertise des techniciens des Agences Territoriales de Développement Agricoles (ATDA) pour garantir une dynamique d'accompagnement aux éleveurs. Des appuis de partenaires techniques privés (cabinets vétérinaires, consultants fermiers, etc.) peuvent s'avérer profitables dans l'information, l'éducation et la formation des éleveurs de porcs sur la l'alimentation, les grandes enzooties et épizooties, et la gestion des troupeaux. Les acteurs scientifiques devront contribuer à l'amélioration de la production par le développement de protocoles de renforcement de l'immunité, de formules alimentaires efficaces mais à moindre coût et approfondir l'investigation sur les contraintes de la filière.

\section{Conclusion}

L'élevage porcin est pratiqué dans toutes les contrées du Bénin le plus souvent suivant un mode extensif. Il est en général de petite taille (quelques têtes) et constitue une activité secondaire pour des fonctionnaires ou des ménagères. Cependant, il génère des bénéficies subséquents et tient une bonne part dans les productions animales. Mais la structure et le fonctionnement de la filière porcine tant au niveau de la production que de la commercialisation souffrent toujours de nombreux défauts ; ce qui a pour conséquence une sous production. L'existence actuelle d'un environnement politique, structurel et financier favorables constitue une véritable opportunité pour lever les contraintes techniques, sanitaires pour faire prospérer la filière porcine. Il reste que des efforts de professionnalisation et de maîtrise zootechnique et sanitaire soient entrepris.

\section{CONFLIT D'INTERETS}

Les auteurs déclarent ne pas avoir de conflit d'intérêts dans la publication de cet article.

\section{CONTRIBUTIONS DES AUTEURS}

WAT a mené l'étude sur le terrain, collecté, analysé, interprété les données et participé à la conception de l'étude. SA a conçu l'étude et supervisé toute l'étude. SS a participé à la correction du manuscrit.

\section{REMERCIEMENTS}

Les auteurs remercient tous les éleveurs et les agents vétérinaires des ATDA, qui ont accepté de contribuer à la réalisation de cette étude et de se rendre disponibles pour les enquêtes. 


\section{RÉFÉRENCES}

Agbokounou AM, Ahounou GS, Youssao AKI, Mensah GA, Koutinhouin B, Hornick JL. 2016. Caractéristiques de l'élevage du porc local d'Afrique. J. Anim. Plant Sci., 30(1) : 4701-4713. http://m.elewa.org/JAPS/2016/29.3/3.Ari stide.pdf

Atodjinou FTR, Dotcho CDG. 2006. Caractéristiques de l'élevage des porcs locaux dans les élevages périurbains de Cotonou et d'Abomey-Calavi. Mémoire pour l'obtention du Diplôme d'études agricoles tropicales, Lycée Meidji de Sékou, Bénin. 80p.

Ayssiwede SB. 2004. La filière porcine au Bénin : production, commercialisation, propositions d'amélioration et perspectives de développement. Thèse de Doctorat en Médecine Vétérinaire. Ecole Inter-Etats des Sciences et de médecine vétérinaire de Dakar, Sénégal, Ecole Inter-Etats des Sciences et de médecine vétérinaire.161p.

Banque Mondiale. 2019. La Banque mondiale au Bénin. Bénin présentation; https://www.banquemondiale.org/fr/coun try/benin/overview, Consulté le 15 Février 2021.

Dahouda M, Amoussa S, Dossa L, Kiki P, Houessou O. 2019. Les stratégies d'utilisation des ressources alimentaires locales par les caprins dans quatre communes du Bénin. Revue Inter. Sci Appl. (RISA), 2 (2) : 23-33.

Direction de l'Elevage (DE). 2017. Performance du sous-secteur élevage : quelques données de 2016. Rapport d'étape. République du Bénin. 5 pp.

Djimenou D, Adoukonou-Sagbadja H, Koudande DO, Chrysostome CAAM, Hounzangbe-Adote SM, Agbangla C. 2017. Caractéristiques sociodémographiques des éleveurs de porcs (Sus Scrofa domesticus) et structure du cheptel porcin au Sud du Bénin. Int. J. Biol. Chem. Sci., 11(5) : 2177-2193.

Dognon SR, Salifou CFA, Dougnon J, Dahouda M, Scippo ML, Youssao AKI. 2018. Production, importation et qualité des viandes consommées au Bénin. $J$. Appl. Biosc., 124: 12476-12487. DOI: https://dx.doi.org/10.4314/jab.v124i1.9

FAOSTAT County. 2016. Consulté le 6 Février 2020.

http://fenix.fao.org/faostat/beta/fr/\#data

FAOSTAT. 2017. Base de données en ligne. http://www.fao.org/faostat/fr/\#data, consulté le 03 novembre 2020.

FAOSTAT. 2020. Base de données en ligne. http://www.fao.org/faostat/fr/\#data, consulté le 03 novembre 2020.

Houndonougbo MF, Adjolohoun S, Aboh BA, Singbo A, Chrysostome CAA. 2012. Caractéristiques du système d'élevage porcin au Sud-Est du Bénin. Bulletin de la Recherche Agronomique du Bénin (BRAB) (Numéro spécial Elevage \& Faune) : 1521.

Kiki PS, Dahouda M, Toleba SS, Ahounou SG, Dotché IO, Govoeyi B, Dehoux JP. 2018. Gestion de l'alimentation des porcs et contraintes de l'élevage porcin au SudBénin. Revue Elev. Méd. Vét. Pays 
Trop., 71(1-2) : $\quad$ 67-74. DOI : https://doi.org/10.19182/remvt.31223

Ministère de l'Economie et des Finances (MEF). 2020. Budget National 2020. Ministère de l'Economie et des Finances. Cotonou, Bénin.

Mopaté LY, Kaboré-Zoungrana C-Y. 2010. Dynamique des élevages et caractéristiques des producteurs de porcs de la ville de N'Djaména, Tchad. In : Actes du colloque « Savanes africaines en développement : innover pour durer », Jamin, JY, Seyni Boukar L, Floret C, (éds) ; 20-23 Avril 2009, Garoua, Cameroun, 9p.

Ndébi G, Kamajou J, Ongla J. 2009. Analyse des contraintes au développement de la production porcine au Cameroun. Tropicultura, 27(2) : 70-76.

Programme d'Actions du Gouvernement (PAG). 2016-2021, 98p.

Soglohoun PY. 2017. Inventaire des pathologies porcines et mesures de biosécurité dans les départements de l'Ouémé et du Plateau. Mémoire de Licence en Production Animale, Université d'Abomey-Calavi, Bénin : 45p. Vignon G. 2015. Typologie des habitats de porcs et proposition d'un modèle pour la biosécurité. Mémoire de Licence Professionnelle en Production et Santé Animales, Université d'Abomey Calavi, Bénin. : 68p.
Youssao AKI, Koutinhouin GB, Kpodekon TM, Bonou AG, Adjakpa A, Ahounou GS, Mourot J. 2009b. Performances zootechniques et aptitudes bouchères des porcs locaux au Sud du Bénin. Bull. Anim. Hlth. Prod. Afr, 57: 73-87. DOI : 10.4314/bahpa.v57i1.44053

Youssao AKI, Koutinhouin GB, Kpodekon TM, Yacoubou A, Bonou AG, Adjakpa A, Taiwo R. 2009a. Amélioration génétique des performances zootechniques du porc local du Bénin par croisement avec le Large White. Int. J. Biol. Chem. Sci., 3(4): 653-662. 10.4314/ijbcs.v3i4.47158

Youssao AKI, Kpodékon TM, Koutinhouin GB, Adjapka A, Yacoubou A, Ahounou S. 2008. Influence de la castration des mâles sur les performances de croissance, les caractéristiques de la carcasse et les qualités de la viande du porc local du Bénin. Bulletin de la Recherche Agronomique du Bénin (BRAB), 61 : 1724.

Youssao AKI. 2007. Cours d'Ethnologie des animaux de productions. Département de Production et Santé Animales/EPAC/ Université Abomey-Calavi, Bénin, 90p.

Youssao AKI. 2015. Programme National d'Amélioration Génétique. Projet d'Appui aux Filières Lait et Viande (PAFILAV). Cotonou (Bénin), 362p. 